\title{
A NEW PROOF OF THE RAN-MATSUSAKA CRITERION FOR JACOBIANS
}

\begin{abstract}
ALBERTO COLLINO
ABSTRACT. We give a characteristic free proof of a criterion which was first proved by Ran over $\mathbf{C}$.
\end{abstract}

THEOREM. Let $X$ be an abelian variety of dimension $n$ over an algebraically closed field. Let $G$ be an effective one-cycle which generates $X$ and let $D$ be an ample divisor on $X$ such that $\operatorname{deg}(D \cdot G)=n$. Then $(X, D, G)$ is a Jacobian triple.

REMARK. Ran's criterion [3] is an extension of Matsusaka's criterion [1]. Ran points out that Matsusaka's main tool is a certain endomorphism $\alpha$; the composite map $f b^{*} r$ in the diagram below is the endomorphism $\alpha(G, D)$ of [1].

We consider first the case where $G$ is reduced and irreducible. Let $a: C \rightarrow G$ be the normalization map, $b: C \rightarrow G \rightarrow X$ be the composite map, $C(n)$ denote the $n$th symmetric product of $C, J$ be the jacobian of $C$ and $D_{x}$ be the translate of $D$ by $x \in X$, i.e. $D_{x}=\{(d+x): d \in D\}$. Our main tool is the diagram

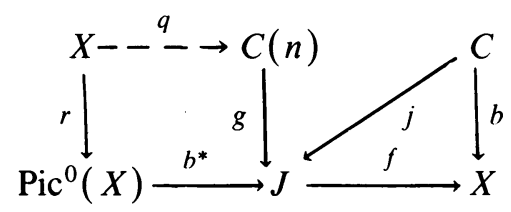

where $r(x)=\operatorname{class}\left(D_{x}-D\right), g\left(p_{1}, p_{2}, \ldots, p_{n}\right)=\operatorname{class}\left(p_{1}+p_{2}+\cdots+p_{n}\right)-$ $b^{*}$ class $D$ and $q$ is defined on the open set $V=\left\{x: G \Varangle D_{x}\right\}$ by the rule $q(x)=b^{*} D_{x}$, as a divisor on $C$. The map $j$ is just the usual Abel-Jacobi morphism and $f$ is induced by the universal property of $J=\operatorname{Alb}(C)$.

Since $D$ is ample, $r$ is an isogeny. $\operatorname{Ker} b^{*}$ is finite because $f$ is surjective and $b^{*}$ is the dual map to $f$. Then $n=\operatorname{dim} X=\operatorname{dim}$ closure $(g q V)$, so that $q V$ is dense in $C(n)$, both having the same dimension. We have $b^{*} r X=g C(n)$, therefore $b^{*} r X=J$, because $g C(n)$ generates $J$ and $b^{*} r$ is a homomorphism. It follows that (i) $n=$ genus $C$, and (ii) $f$ is an isogeny.

The divisor $E=f^{*} D$ is ample on $J$. Further we have

LEMMA. $E$ induces a principal polarization on $J$; hence $\operatorname{deg}\left(E^{n}\right)=n$ !.

Received by the editors May 20, 1983 and, in revised form, November 1, 1983.

1980 Mathematics Subject Classification. Primary 14K05, 14H40.

${ }^{1}$ Supported in part by a grant from the Italian Ministry of Public Education and the C.N.R. 
Proof. By [2, III, 16] it suffices to show $\operatorname{dim}|E|=0$, i.e. that $E$ is linearly isolated. If $E$ is not linearly isolated, neither are its translates. Let $F=E_{y}$ be a general translate and $H \neq F$ a divisor which is linearly equivalent to $F$. It is easy to see that $C \cdot F$ and $C \cdot H$ are different divisors on $C$; hence $C \cdot F$ is a special divisor of degree $n$. Now $F=E_{y}=f^{*} D_{x}, x=f(y)$ and $C \cdot F=b^{*} D_{x}=q(x)$; therefore, for general $x, q(x)$ is a special divisor. This is a contradiction because $q V$ is dense in $C(n)$.

Proposition. $f: J \rightarrow X$ is an isomorphism.

Proof. $n !=\operatorname{deg}\left(E^{n}\right)=\operatorname{deg}\left(f^{*} D\right)^{n}=\operatorname{deg}(f) \operatorname{deg}\left(D^{n}\right)$ and then $\operatorname{deg}(f)=1$, because $\operatorname{deg}\left(D^{n}\right) / n !=\chi(O(D))$.

We identify $J$ and $X$ via $f$; hence $G=C$.

To finish we show that $-D=\{-d: d \in D\}$ is a translate of the standard image $U$ of $C(n-1)$ in $J$, via the Abel-Jacobi map.

Let $\alpha: C(n) \rightarrow J$ be $\alpha=r^{-1}\left(b^{*}\right)^{-1} g, \alpha$ is well defined because $r$ is an isomorphism, since $D$ induces a principal polarization. The map $q$ is the inverse of $\alpha$; hence for the general point $\left(p_{1}, \ldots, p_{n}\right)$ of $C(n)$ we have $\alpha\left(p_{1}, \ldots, p_{n}\right)=x$, with $D_{x} \cdot C=p_{1}+$ $\cdots+p_{n}$.

Let $\beta$ : $C \times C(n-1) \rightarrow J$ be given by $\beta\left(y ; p_{2}, \ldots, p_{n}\right)=y-\alpha\left(y, p_{2}, \ldots, p_{n}\right)$, where $y$ denotes both the point on $C$ and its image in $J$.

LEMMA. $D=$ Image $\beta$.

Proof. If $\left(y, p_{2}, \ldots, p_{n}\right)$ is a general point in $C(n)$, then $\alpha\left(y, p_{2}, \ldots, p_{n}\right)=x$, with $D_{x} \cdot C=y+p_{2}+\cdots+p_{n}$ and hence $y \in D_{x}$, i.e. $(y-x) \in D$. It follows Image $\beta$ $\subseteq D$. Let $D^{\prime \prime}=\operatorname{closure}(D-$ Image $\beta)$, we show $D^{\prime \prime}=\varnothing$. If $D^{\prime \prime}$ is not empty it is a divisor in $J$. A general translate of $C$ intersects $D^{\prime \prime}$ and Image $\beta$ properly and outside of their intersection. Without restriction we may assume this translate to be $C$ itself. Let $C \cdot D=w+q_{2}+\cdots+q_{n}$ with $w \in D^{\prime \prime}$. Then $\beta\left(w ; q_{2}, \ldots, q_{n}\right)=w$, a contradiction.

Up to a translation of $D$ we may assume that $0 \in$ Image $\beta$. By the rigidity lemma [2, II, 4] there are morphisms $\gamma: C \rightarrow J$ and $\delta: C(n-1) \rightarrow J$ such that $\beta\left(y ; p_{2}, \ldots, p_{n}\right)=\gamma(y)+\delta\left(p_{2}, \ldots, p_{n}\right)$. Fixing $y$ we notice that

$$
\operatorname{dim} \beta(\{y\} \times C(n-1))=(n-1),
$$

because $\alpha$ is generically injective and hence $\beta(\{y\} \times C(n-1))=D$ for all $y$. It follows that $\gamma(C)=\{0\}$, because $D$ is not left fixed by any translation; hence

$$
\delta\left(p_{2}, \ldots, p_{n}\right)=\beta\left(y ; p_{2}, \ldots, p_{n}\right)=y-\alpha\left(y, p_{2}, \ldots, p_{n}\right) \quad \forall y \in C .
$$

Fix now $n-1$ points, $z_{1}, \ldots, z_{n-1}$, on $C$. For any point $\left(p_{1} ; p_{2}, \ldots, p_{n}\right)$ of $C \times C(n-1)$ one has

$$
\begin{aligned}
& \beta\left(p_{1} ; p_{2}, \ldots, p_{n}\right)=\delta\left(p_{2}, \ldots, p_{n}\right)=-\alpha\left(z_{1}, p_{2}, \ldots, p_{n}\right)+z_{1} \\
& \quad=-p_{2}+\delta\left(p_{3}, \ldots, p_{n}, z_{1}\right)+z_{1}=-p_{2}-p_{3}+\delta\left(p_{4}, \ldots, p_{n}, z_{1}, z_{2}\right)+z_{1}+z_{2} \\
& \quad=\cdots=-\left(p_{2}+\cdots+p_{n}\right)+\left(\delta\left(z_{1}, z_{2}, \ldots, z_{n-1}\right)+z_{1}+z_{2}+\cdots+z_{n-1}\right) .
\end{aligned}
$$

Since $D=\beta(C \times C(n-1)), D$ is a translate of $-U$, where $U=\left\{\left(y_{1}+\cdots+y_{n-1}\right)\right.$ : $\left.y_{i} \in C\right\}$. 
Next one should deal with the case when $G$ is not reduced and irreducible. In a first draft of this paper we sketched the proof, which follows exactly the same pattern as in the preceding case. Following the referee's advice we omit it because it is a rather straightforward exercise, cf. p. 469 in [3].

REMARK. The theorem is still true if the apparently weaker hypothesis $\operatorname{deg}(D \cdot G)$ $\leqslant n$ was given instead of the equality. The proof is the same.

\section{REFERENCES}

1. T. Matsusaka, On a characterization of a Jacobian variety, Mem. Colloq. Sci. Kyoto Ser. A 23 (1959), 1-19.

2. D. Mumford, Abelian varieties, Oxford Univ. Press, London and New York, 1970.

3. Z. Ran, On subvarieties of abelian varieties, Invent. Math. 62 (1981), 459-479.

Dipartimento di Matematica, Universita di Torino, 10123 Torino, ITAly 